\title{
SZEGÖ LIMIT THEOREMS ON THE SIERPIŃSKI GASKET
}

\author{
KASSO A. OKOUDJOU, LUKE G. ROGERS, AND ROBERT S. STRICHARTZ

\begin{abstract}
We use the existence of localized eigenfunctions of the Laplacian on the Sierpiński gasket (SG) to formulate and prove analogues of the strong Szegö limit theorem in this fractal setting. Furthermore, we recast some of our results in terms of equally distributed sequences.
\end{abstract}

\section{INTRODUCTION}

Let $P_{n}$ be the orthogonal projection of $L^{2}([0,2 \pi)$ onto the linear subspace spanned by the functions $\left\{e^{i m \theta}: 0 \leq m \leq n ; 0 \leq \theta<2 \pi\right\}$. For any function $f$ defined on $[0,2 \pi)$, let $[f]$ be the linear operator corresponding to multiplication by $f$.

In 1952, G. Szegö proved that for a positive function $f \in \mathcal{C}^{1+\alpha}$ where $\alpha>0$, the following holds

$$
\lim _{n \rightarrow \infty} \frac{1}{n+1} \log \operatorname{det} P_{n}[f] P_{n}=\frac{1}{2 \pi} \int_{0}^{2 \pi} \log f(\theta) d \theta .
$$

Equivalently, (1) can be expressed as

$$
\lim _{n \rightarrow \infty} \frac{1}{n+1} \text { Trace } \log P_{n}[f] P_{n}=\frac{1}{2 \pi} \int_{0}^{2 \pi} \log f(\theta) d \theta .
$$

The above result is known as the strong Szegö limit theorem and we refer to [6, 19] for more details and related results.

Today, the strong Szegö limit theorem can be proved under much weaker conditions on $f$, see e.g., [5, 8, 9]. For high dimensional extensions of the strong Szegö limit theorem, we refer to [13] and the references therein.

Date: November 6, 2018.

2000 Mathematics Subject Classification. Primary 35P20, 28A80; Secondary 42C99, 81Q10.

Key words and phrases. Analysis on Fractals, equally distributed sequences, Laplacian, localized eigenfunctions, Sierpiński gasket, strong Szegö limit theorem.

Research of the third author supported in part by the National Science Foundation, grant DMS-065440. 
In fact, the strong Szegö limit theorem is a special case of a more general result proved by Szegö using the fact that $P_{n}[f] P_{n}$ is a Toeplitz form [6. More specifically, let $f$ be a real-valued integrable function such that $m \leq f(x) \leq M$. Then the eigenvalues $\left\{\lambda_{k}^{(n)}\right\}_{k=1}^{n+1}$ of $P_{n}[f] P_{n}$ are contained in $[m, M]$. For any continuous function $F$ defined on this interval, it was proved in [6, Section 5.3] that

$$
\lim _{n \rightarrow \infty} \frac{1}{n+1} \sum_{k=1}^{n+1} F\left(\lambda_{k}^{(n)}\right)=\frac{1}{2 \pi} \int_{0}^{2 \pi} F(f(x)) d x .
$$

Notice that (1) is a specific case of (3), when $F(x)=\log x$. It follows from (3) that the bounded sequences $\left\{\lambda_{k}^{(n+1)}\right\}_{k=1}^{n+1}$ and $\left\{f\left(\frac{2 k \pi}{n+1}\right)\right\}_{k=1}^{n+1}$ are equally distributed in the interval $[m, M]$; see [6, Chapter 5].

By noticing that $e^{i m \theta}$ is an eigenfunction of $\Delta=\frac{d^{2}}{d x^{2}}$, one can view the above results as special cases of Szegö limit theorem for the LaplaceBeltrami operator (or more generally for pseudodifferential operators) on manifolds [7, 12, 13, 21].

In the present paper, we prove the analogue of the strong Szegö limit theorem on the Sierpiński gasket (SG). This set is an example of fractal on which a well established theory of Laplacian exists [1, 10, 16, 18]. In this fractal setting, the non-periodicity of the eigenfunctions of the Laplacian implies that the analogue of the matrix $P_{j}[f] P_{j}$ is no longer related to a Toeplitz form. Thus our results no longer follow from any known proof of (1), but rather rely on the existence of localized eigenfunctions for the Laplacian on SG [2].

The paper is organized as follows: In Section 2 we briefly introduce some key notions from analysis on fractals and give a precise description of the Dirichlet spectrum of the Laplacian on SG. In Section 3 we prove a special case of the strong Szegö theorem that we use in Section 4 to prove an analogue of (1). Finally, Section 5 contains a further extension of the strong Szegö limit theorem on SG.

\section{ANALYSIS ON THE SIERPIŚKI GASKET}

2.1. Basic features. In this section we collect some key facts from analysis on SG that we need to state and prove our results. These come from Kigami's theory of analysis on fractals, and may be found in [10]. An elementary exposition may be found in [16, 18].

Let $F_{1}, F_{2}$ and $F_{3}$ be the contractions defined on $\mathbb{R}^{2}$ respectively by $F_{1}(x)=\frac{1}{2} x, F_{2}(x)=\frac{1}{2} x+\left(\frac{1}{2}, 0\right)$ and $F_{3}(x)=\frac{1}{2} x+\left(\frac{1}{4}, \frac{\sqrt{3}}{4}\right)$. The Sierpiński gasket denoted by $\mathrm{SG}$ is the unique nonempty compact subset of $\mathbb{R}^{2}$ such that $\mathrm{SG}=\cup_{i=1}^{3} F_{i}(\mathrm{SG})$. Alternatively, $\mathrm{SG}$ can be defined 
as a limit of graphs. For a word $\omega=\left(\omega_{1}, \omega_{2}, \ldots, \omega_{m}\right)$ of length $m$, the set $F_{\omega}(\mathrm{SG})=F_{\omega_{1}} \circ \cdots \circ F_{\omega_{m-1}} \circ F_{\omega_{m}}(\mathrm{SG})$ with $\omega_{i} \in\{1,2,3\}$, is called an $m$-cell. Let $V_{0}=\left\{(0,0),(1,0),\left(\frac{1}{2}, \frac{\sqrt{3}}{2}\right)\right\}$, be the boundary of SG and $V_{n}=\cup_{i=1}^{3} F_{i} V_{n-1}, n \geq 1$. Define a sequence of graphs $\Gamma_{m}$ with vertices in $V_{m}$ and edge relation $x \sim_{m} y$ given inductively by: $\Gamma_{0}$ is the complete graph with vertices in $V_{0}$, and $x \sim_{m} y$ if and only if $x$ and $y$ belong to the same $m$-cell $F_{\omega}(\mathrm{SG})$.

In all that follows, we assume that $\mathrm{SG}$ is equipped with the probability measure $\mu$ that assigns the measure $3^{-m}$ to each $m$-cell. We will also need the energy or Dirichlet form that is naturally defined on SG and denoted $\mathcal{E}$. The precise definition of $\mathcal{E}$ will not be given here but can be found in [10, 18]. All we will need in the sequel is that $\mathcal{E}$ gives rise to a natural distance on $\mathrm{SG}$ called the effective resistance metric on $\mathrm{SG}$, and defined for $x, y \in \mathrm{SG}$ by

$$
d(x, y)=(\min \{\mathcal{E}(u, u): u(x)=0 \text { and } u(y)=1\})^{-1} .
$$

It is known that $d(x, y)$ is bounded above and below by constant multiples of $|x-y|^{\log (5 / 3) / \log 2}$, where $|x-y|$ is the Euclidean distance.

For any integer $N>1$ we will consider a partition of the Sierpiński gasket (SG) into

$$
\mathrm{SG}=\cup_{|\omega|=N} F_{\omega} \mathrm{SG},
$$

where for each word $\omega$ of length $N, F_{\omega}(\mathrm{SG})$ is an $N$-cell. Note that there are exactly $3^{N}$ such cells, each of which has a measure $3^{-N}$. If $u$ is a function having support entirely contained in a single $N$-cell then we will say that $u$ is localized at scale $N$.

2.2. The Laplacian and its spectrum. A Laplacian can be defined on SG either through a weak formulation using the energy and measure or as a renormalized limit of graph Laplacians in the following manner [10, 18]. Define the graph Laplacian $\Delta_{m}$ on $\Gamma_{m}$ by

$$
\Delta_{m} f(x)=\sum_{y \sim_{m} x} f(y)-4 f(x)
$$

for $x \in V_{m} \backslash V_{0}$. The Laplacian on SG can now be defined by

$$
\Delta=\frac{3}{2} \lim _{m \rightarrow \infty} 5^{m} \Delta_{m}
$$

A complete description of the spectrum of $\Delta$ on SG was given in [4] using the method of spectral decimation introduced in [15], and a description of the eigenfunctions was obtained by this method in [3], see also [20]. In a nutshell, the spectral decimation method completely 
determines the eigenvalues and the eigenfunctions of $\Delta$ on SG from the eigenvalues and eigenfunctions of the graph Laplacians $\Delta_{m}$. More specifically, for every Dirichlet eigenvalue $\lambda$ of $\Delta$ on SG, there exists an integer $j \geq 1$, called the generation of birth, such that if $u$ is a $\lambda$-eigenfunction and $k \geq j$ then $\left.u\right|_{V_{k}}$ is an eigenfunction of $\Delta_{k}$ with eigenvalue $\gamma_{k}$. The only possible initial values $\gamma_{j}$ are 2, 5 and 6, and subsequent values can be obtained from

$$
\gamma_{k}=\frac{1}{2}\left(5+\epsilon_{k} \sqrt{25-4 \gamma_{k-1}}\right) \text { for } k>j
$$

where $\epsilon_{k}$ can take the values \pm 1 . The sequence $\gamma_{k}$ is related to $\lambda$ by

$$
\lambda=\frac{3}{2} \lim _{k \rightarrow \infty} 5^{k} \gamma_{k}
$$

In particular the convergence of (9) implies that $\epsilon_{k}=1$ for at most a finite number of $k$ values. We let $l=\min \left\{k: \epsilon_{k}=-1\right\}$ and call it the generation of fixation.

An interesting and useful feature is that there are a great many eigenfunctions which satisfy both Dirichlet and Neumann boundary conditions; this is a general property of the Laplacian on fractals with sufficient symmetry [2, and in the case of SG it implies both that most eigenfunctions are localized on small sets, and that eigenspaces have high multiplicity.

Using the spectral decimation algorithm and elementary properties of the map in (8) one can see that the size of an eigenvalue depends (up to constant factors) on its generation of fixation, and its multiplicity depends on its generation of birth. We summarize the relevant features of eigenvalues at the bottom of the spectrum and their eigenspaces in Proposition 1 below, and refer the reader to the original treatments [2, 3, 4], or the exposition in [18] for proofs.

Proposition 1. There is a constant $\kappa$ such that the $\frac{1}{2}\left(3^{m+1}-3\right)$ smallest eigenvalues of $-\Delta$ are precisely those with size at most $\kappa 5^{m}$, and all have generation of fixation $l \leq m$. The eigenvalues, their multiplicities, and bases for their eigenspaces may be described as follows.

- The 2-series eigenvalues are those obtained from (8) and (9) with generation of birth $j=1$ and $\gamma_{j}=2$. Each such eigenvalue has multiplicity 1.

- The 5-series eigenvalues are those obtained from (8) and (9) with any generation of birth $j \geq 1$ and $\gamma_{j}=5$. There are $2^{m-j}$ such eigenvalues for each $1 \leq j \leq m$, every one having multiplicity $\frac{1}{2}\left(3^{j-1}+3\right)$. For each such eigenvalue, there is a basis for the corresponding eigenspace in which all but two of 
the basis functions have support in a collection of $(j-1)$-cells arranged in a loop around a "hole" of scale at least $(j-1)$ in $S G$. There are $\frac{1}{2}\left(3^{j-1}-1\right)$ such holes and one eigenfunction for each hole.

- The 6-series eigenvalues are those obtained from (8) and (9) with any generation of birth $j \geq 2, \gamma_{j}=6$ and $\epsilon_{j+1}=+1$. There are $2^{m-j-1}$ such eigenfunctions for each $2 \leq j<m$ and 1 for $j=m$, every one having multiplicity $\frac{1}{2}\left(3^{j}-3\right)$. For each such eigenvalue there is a basis for the corresponding eigenspace that is indexed by points of $V_{j-1} \backslash V_{0}$, and in which each basis element is supported on the union of the two $j$-cells that intersect at the corresponding point in $V_{j-1} \backslash V_{0}$.

Recall that a function is said to be localized at scale $N$ if its support is contained entirely in a single $N$-cell. It is apparent from the above that there are eigenfunctions that are localized at scale $N$ provided $j>N$. For later use, we compute the number of these that occur in each of the 5 and 6 -series.

Let $j>N$ and consider a 6 -series eigenvalue with generation of birth $j$. In the associated eigenspace there are $\frac{1}{2}\left(3^{N+1}-3\right)$ basis functions corresponding to the vertices in $V_{N} \backslash V_{0}$, and which are not localized at scale $N$. The remaining $\frac{1}{2}\left(3^{j}-3^{N+1}\right)$ basis functions correspond to vertices in $V_{j-1} \backslash V_{N}$ and are localized at scale $N$.

For $j>N$ and a fixed 5-series eigenvalue, the basis elements for the eigenspace are supported on loops or chains. A loop is contained in a cell of scale $N$ if and only if the hole it encircles has scale at least $N+1$. The number of holes of scale at most $N$ is $\frac{1}{2}\left(3^{N}-1\right)$, so the number of basis elements not localized at scale $N$ is this plus the two that are not loops, giving $\frac{3^{N}}{2}$ in total. The remaining $\frac{1}{2}\left(3^{j-1}-3^{N}\right)$ basis eigenfunctions are localized at scale $N$.

\section{Szegö limit theorem on SG For a Single Eigenspace}

In this section we prove a Szegö limit theorem for a single 5-series or 6-series eigenspace of the Laplacian on SG.

Let $\lambda_{j}$ be a 6 -series eigenfunction with generation of birth $j$ and eigenspace $E_{j}$. Denote the span of those eigenfunctions corresponding to $\lambda_{j}$ that are localized at scale $N<j$ by $E_{j}^{N}$. Let $d_{j}^{N}=\operatorname{dim} E_{j}^{N}=$ $\frac{1}{2}\left(3^{j}-3^{N+1}\right)$ and $\alpha_{j}^{N}=\frac{1}{2}\left(3^{N+1}-3\right)$ be the dimension of the complementary space in $E_{j}$. Since there are $3^{N}$ cells of scale $N$, we see that the number of eigenfunctions supported on a single cell is $m_{j}^{N}=$ $\frac{1}{2}\left(3^{j-N}-3\right)$. 
An analogous construction may be done for a 5-series eigenfunction, with the only change being that in this case $\alpha_{j}^{N}=\frac{3^{N}-3}{2}, d_{j}^{N}=\frac{1}{2}\left(3^{j-1}-\right.$ $\left.3^{N}\right)$ and $m_{j}^{N}=\frac{1}{2}\left(3^{j-N-1}-1\right)$.

For each $N$-cell, use the Gram-Schmidt process to orthonormalize the collection of eigenfunctions supported on that cell. Since functions on separate cells are already orthogonal, taking the union over all $N$-cells gives an orthonormal basis $\left\{\tilde{u}_{k}\right\}_{k=1}^{d_{j}^{N}}$ for $E_{j}^{N}$. Adjoining the remaining basis elements of $E_{j}$ and again using the Gram-Schmidt process extends this to an orthonormal basis

$$
\left\{u_{k}\right\}_{k=1}^{d_{j}}=\left\{\tilde{u}_{k}\right\}_{k=1}^{d_{j}^{N}} \cup\left\{v_{k}\right\}_{k=1}^{\alpha_{j}^{N}},
$$

for $E_{j}$, where only the $v_{k}$ are not localized at scale $N$.

Let $P_{j}$ be the projection of $L^{2}(\mathrm{SG})$ onto $E_{j}$. For $g \in L^{2}(\mathrm{SG}), P_{j}$ is defined by

$$
P_{j} g(x):=\sum_{k=1}^{d_{j}} g_{k} u_{k}(x)=\sum_{k=1}^{d_{j}}\left\langle g, u_{k}\right\rangle u_{k}(x) .
$$

For a real-valued function $f$ on $\mathrm{SG}$, we recall that $[f]$ the operator corresponding to the pointwise multiplication by $f$.

\subsection{The case of simple functions.}

Lemma 1. Let $f=\sum_{k=1}^{3^{N}} a_{k} \chi_{C_{k}}$ where we assume that $a_{k}>0$ for all $k$ and $\chi_{C_{k}}$ denotes the characteristic function of the $N$-cell $C_{k}$. Then for $P_{j}$ as above,

$$
\lim _{j \rightarrow \infty} \frac{1}{d_{j}} \log \operatorname{det} P_{j}[f] P_{j}=\int_{S G} \log f(x) d \mu(x) .
$$

Furthermore, for $j$ large enough,

$$
\frac{1}{d_{j}} \log \operatorname{det} P_{j}[f] P_{j}-\int_{S G} \log f(x) d \mu(x)=O\left(d_{j}^{-1}\right) .
$$

Proof. For $j>N$ set $M_{j}=P_{j}[f] P_{j}$. Then $M_{j}$ is a $d_{j} \times d_{j}$ matrix which has block structure

$$
M_{j}=\left[\begin{array}{cc}
R_{j} & \star \\
0 & N_{j}
\end{array}\right]
$$

with respect to the basis $\left\{u_{k}\right\}_{k=1}^{d_{j}}$. Here $R_{j}$ is an invertible $d_{j}^{N} \times d_{j}^{N}$ matrix corresponding to the "localized" part, while $N_{j}$ is an invertible $\alpha_{j}^{N} \times \alpha_{j}^{N}$ matrix corresponding to the "non-localized" part. Furthermore, $R_{j}$ is a block diagonal matrix, where each block is an $m_{j}^{N} \times m_{j}^{N}$ matrix that corresponds to a single $N$-cell $C_{k}$, and is therefore simply 
$a_{k} I_{m_{j}^{N}}$ where $I_{d}$ is notation for the $d \times d$ identity matrix. It follows immediately that

$$
\begin{aligned}
\log \operatorname{det} M_{j} & =\log \operatorname{det} R_{j}+\log \operatorname{det} N_{j} \\
& =\log \left(\prod_{k=1}^{3^{N}} a_{k}^{m_{j}^{N}}\right)+\log \operatorname{det} N_{j} \\
& =m_{j}^{N}\left(\sum_{k=1}^{3^{N}} \log a_{k}\right)+\log \operatorname{det} N_{j} .
\end{aligned}
$$

Using $d_{j}^{N}=3^{N} m_{j}^{N}$ we conclude

$$
\begin{aligned}
\frac{1}{d_{j}} \log \operatorname{det} M_{j} & =\frac{m_{j}^{N} 3^{N}}{d_{j}} \sum_{k=1}^{3^{N}} 3^{-N} \log a_{k}+\frac{1}{d_{j}} \log \operatorname{det} N_{j} \\
& =\frac{d_{j}^{N}}{d_{j}} \int_{\mathrm{SG}} \log f(x) d \mu(x)+\frac{1}{d_{j}} \log \operatorname{det} N_{j},
\end{aligned}
$$

and since $d_{j}-d_{j}^{N}=\alpha_{j}^{N}$, we have

$$
\begin{aligned}
& \frac{1}{d_{j}} \log \operatorname{det} M_{j}-\int_{\mathrm{SG}} \log f(x) d \mu(x) \\
& =\frac{-\alpha_{j}^{N}}{d_{j}} \int_{\mathrm{SG}} \log f(x) d \mu(x)+\frac{1}{d_{j}} \log \operatorname{det} N_{j} .
\end{aligned}
$$

We can now afford a crude estimate of the term $\log \operatorname{det} N_{j}$. Since $f \in L^{\infty}$, the multiplier $[f]$ is bounded on $L^{2}$ by $\|f\|_{\infty}$. It follows that $\left\langle N_{j} g, g\right\rangle \leq\|f\|_{\infty}$ for any $g \in E_{j}$ with $\|g\|_{2}=1$, and therefore that $\operatorname{det} N_{j} \leq\|f\|_{\infty}^{\alpha_{j}^{N}}$. Combining this with (12) we see

$$
\left|\frac{1}{d_{j}} \log \operatorname{det} M_{j}-\int_{\mathrm{SG}} \log f(x) d \mu(x)\right| \leq \frac{\alpha_{j}^{N}}{d_{j}}\left(\|\log f(x)\|_{1}+\|f\|_{\infty}\right)
$$

which completes the proof because $\alpha_{j}^{N}$ is bounded by a constant multiple of $3^{N}$ and $d_{j}$ is comparable to $3^{j}$.

\subsection{The case of continuous positive functions.}

Theorem 1. Let $f$ be a positive and continuous function on $S G$. Then

$$
\lim _{j \rightarrow \infty} \frac{1}{d_{j}} \log \operatorname{det} P_{j}[f] P_{j}=\int_{S G} \log f(x) d \mu(x) .
$$

If in addition, we assume that $f$ is Hölder continuous of order $\alpha$ in the resistance metric $R$ on $S G$, then

$$
\frac{1}{d_{j}} \log \operatorname{det} P_{j}[f] P_{j}-\int_{S G} \log f(x) d \mu(x)=O\left(d_{j}^{-\beta}\right)
$$


where

$$
\beta=\frac{\alpha \log (5 / 3)}{\log 3+\alpha \log (5 / 3)}=1-\frac{\log 3}{\alpha \log (5 / 3)+\log 3} .
$$

Proof. Since SG is compact, $\min _{x \in \mathrm{SG}} f(x)=m>0$. Given $\epsilon>0$, uniform continuity provides $N$ and a simple function $f_{N}=\sum_{k=1}^{3^{N}} a_{k} \chi_{C_{k}}$ such that

$$
\left\|f-f_{N}\right\|_{\infty}<\min \left(\frac{1}{2}, \frac{m}{2}, \frac{\epsilon m}{2}\right)
$$

from which the following are immediate,

$$
\begin{gathered}
\frac{\left|f(x)-f_{N}(x)\right|}{\left|f_{N}(x)\right|} \leq \epsilon, \\
1-\epsilon \leq \frac{f(x)}{f_{N}(x)} \leq 1+\epsilon \\
-2 \epsilon \leq \log (1-\epsilon) \leq \log \left(\frac{f(x)}{f_{N}(x)}\right) \leq \log (1+\epsilon) \leq \epsilon .
\end{gathered}
$$

Note that (16) implies $\left|\int_{S G} \log f-\int_{S G} \log f_{N}\right| \leq 2 \epsilon$.

Now let us estimate $\log \operatorname{det} P_{j}[f] P_{j}$ in the same manner as was done in Lemma 1. It has a block structure like (11), but the diagonal blocks in $R_{j}$ are no longer multiples of the identity matrix $I_{m_{j}^{N}}$. However it follows from (15) that the values on the diagonal corresponding to $C_{k}$ are bounded below by $a_{k}(1-\epsilon)$ and above by $a_{k}(1+\epsilon)$, and thus

$$
\frac{d_{j}^{N}}{d_{j}} \log (1-\epsilon) \leq \frac{1}{d_{j}} \log \operatorname{det} R_{j}-\frac{d_{j}^{N}}{d_{j}} \int_{\mathrm{SG}} f_{N} d \mu \leq \frac{d_{j}^{N}}{d_{j}} \log (1+\epsilon)
$$

and in particular $\left|d_{j}^{-1} \log \operatorname{det} R_{j}-d_{j}^{N} d_{j}^{-1} \int_{S G} f_{N}\right| \leq 2 \epsilon$.

Combining these estimates with the same $\log \operatorname{det} N_{j} \leq \alpha_{j}^{N}\|f\|_{\infty}$ bound used in Lemma 1 we have

$$
\begin{aligned}
& \left|\frac{1}{d_{j}} \log \operatorname{det} P_{j}[f] P_{j}-\int_{S G} \log f d \mu\right| \\
& \leq\left|\frac{1}{d_{j}} \log \operatorname{det} R_{j}-\frac{d_{j}^{N}}{d_{j}} \int_{S G} \log f_{N} d \mu\right|+\frac{\alpha_{j}^{N}}{d_{j}}\left|\int_{S G} \log f_{N} d \mu\right| \\
& \quad+\left|\int_{S G} \log f_{N}-\log f d \mu\right|+\frac{1}{d_{j}} \log \operatorname{det} N_{j} \\
& \leq 4 \epsilon+c \frac{\alpha_{j}^{N}}{d_{j}}\left(\|f\|_{\infty}+\|\log f\|_{1}\right) \\
& \leq 4 \epsilon+c 3^{N-j}\left(\|f\|_{\infty}+\|\log f\|_{1}\right) .
\end{aligned}
$$

This gives the first statement of the theorem.

In the case that $f$ is Hölder continuous of order $\alpha$ in the resistance metric we see that

$$
\epsilon=\left\|f-f_{N}\right\|_{L^{\infty}}=O\left(\left(\frac{3}{5}\right)^{N \alpha}\right) .
$$


For a fixed large $j$ we may then choose $N$ such that the bound in (17) is minimized, which occurs when $\epsilon \approx 3^{N-j}$. Setting $(3 / 5)^{N \alpha}=3^{N-j}$ we compute

$$
3^{N-j}=3^{\frac{-j \alpha \log (5 / 3)}{\log 3+\alpha \log (5 / 3)}}
$$

and substitute into (17) to obtain (14), using $d_{j} \approx 3^{j}$.

Remark 1. The following special cases of (14) are worth pointing out. If $\alpha=1 / 2$ (which is the case when $f \in \operatorname{dom\mathcal {E}}$ ), then $\beta=1-\frac{\log 9}{\log 15}$.

If $\alpha=1$ (which is the case if $f \in \operatorname{dom} \Delta$ ), then $\beta=1-\frac{\log 3}{\log 5}$.

\section{General Szegö Theorem on SG}

In this section we prove analogues of the results proved in Section 3 for the situation where we look at all eigenvalues up to a certain value $\Lambda$. We therefore let $E_{\Lambda}$ be the span of all eigenfunctions corresponding to eigenvalues $\lambda$ of $-\Delta$ for which $\lambda \leq \Lambda$, let $P_{\Lambda}$ be projection onto $E_{\Lambda}$, and set $d_{\Lambda}=\operatorname{dim}\left(E_{\Lambda}\right)$. We also suppose that a scale $N$ is fixed.

Since $-\Delta$ is self-adjoint the eigenspaces of distinct eigenvalues are orthogonal. For each $\lambda<\Lambda$ from either the 5 -series or the 6 -series, and having generation of birth $j>N$ we take an orthonormal basis for the corresponding eigenspace of the type described in Section 3. For all other eigenspaces in $E_{\Lambda}$ we simply take orthonormal bases. The union of the basis vectors is then a basis for $E_{\Lambda}$ and in this basis the operator $P_{\Lambda}[f] P_{\Lambda}$ is a block diagonal matrix $M_{\Lambda}$ with one block $M_{\lambda}$ for each eigenvalue $\lambda \leq \Lambda$.

Theorem 2. Let $f>0$ be a continuous function on $S G$. Then,

$$
\lim _{\Lambda \rightarrow \infty} \frac{1}{d_{\Lambda}} \log \operatorname{det} M_{\Lambda}=\int_{S G} \log f(x) d \mu(x) .
$$

If in addition we assume that $f$ is Hölder continuous of order $\alpha$ in the resistance metric $R$ on $S G$ then

$$
\frac{1}{d_{\Lambda}} \log \operatorname{det} M_{\Lambda}-\int_{S G} \log f(x) d \mu(x)=O\left(d_{\Lambda}^{-\tilde{\beta}}\right)
$$

where

$$
\tilde{\beta}=\beta\left(1-\frac{\log 2}{\log 3}\right)=\left(\frac{\alpha \log (5 / 3)}{\log 3+\alpha \log (5 / 3)}\right)\left(1-\frac{\log 2}{\log 3}\right) .
$$

Proof. Fix $\epsilon>0$. It is clear that $\log \operatorname{det} M_{\Lambda}=\sum_{\lambda<\Lambda} \log \operatorname{det} M_{\lambda}$. If $\lambda$ is one of the 5 or 6 -series eigenvalues with $j>N$ then replacing $f$ by $f_{N_{0}}$ as in the proof of Theorem 1 we have from (17)

$$
\left|\log \operatorname{det} M_{\lambda}-d_{\lambda} \int_{S G} \log f d \mu\right| \leq 4 \epsilon d_{\lambda}+c 3^{N}\left(\|f\|_{\infty}+\|\log f\|_{1}\right) \text {. }
$$


Now we let $\Gamma_{N}$ be the set of $\lambda<\Lambda$ with generation of birth $j>N$ and sum (20) over $\lambda \in \Gamma_{N}$, noting that $d_{\Lambda}^{-1} \sum_{\lambda \in \Gamma_{N}} d_{\lambda} \leq 1$. Using the trivial bound $\log \operatorname{det} M_{\lambda} \leq d_{\lambda}\|f\|_{\infty}$ for the remaining terms in log $\operatorname{det} M_{\Lambda}$, and $d_{\lambda}\|\log f\|_{1}$ for those making up the integral, we obtain

$$
\begin{aligned}
& \left|\frac{1}{d_{\Lambda}} \log \operatorname{det} M_{\Lambda}-\int_{S G} \log f d \mu\right| \\
& \quad \leq 4 \epsilon+\left(\|f\|_{\infty}+\|\log f\|_{1}\right)\left(\left(\frac{c 3^{N}}{d_{\Lambda}} \sum_{\lambda \in \Gamma_{N}} 1\right)+\left(\sum_{\lambda \notin \Gamma_{N}} \frac{d_{\lambda}}{d_{\Lambda}}\right)\right) .
\end{aligned}
$$

The remaining work in the proof is to estimate the number of eigenvalues in $\Gamma_{N}$ and the sum of the dimensions $d_{\lambda}$ for $\lambda \notin \Gamma_{N}$, which we do using Proposition 1. For this purpose, take $m \in \mathbb{N}$ so $\kappa 5^{m-1} \leq$ $\Lambda<\kappa 5^{m}$, where $\kappa$ is as in Proposition 1, Since $\Gamma_{N}$ is empty and the estimate is trivial if $N \geq m$, we assume without loss of generality that $N<m$. The eigenvalues less than $\kappa 5^{m}$ and having generation of birth $j \leq N$ number $2^{m-1}$ with multiplicity 1 from the 2 -series, $2^{m-j}$ with multiplicity $\frac{1}{2}\left(3^{j-1}+3\right)$ from the 5 -series and $2^{m-j-1}$ with multiplicity $\frac{1}{2}\left(3^{j}-3\right)$ if $2 \leq j \leq N$. Summing these gives

$$
2^{m-1}+\sum_{1}^{N} 2^{m-j-1}\left(3^{j-1}+3\right)+\sum_{2}^{N} 2^{m-j-2}\left(3^{j}-3\right)=O\left(2^{m-N} 3^{N}\right) .
$$

SO

$$
\sum_{\left\{\lambda \notin \Gamma_{N}: \lambda \leq \Lambda\right\}} d_{\lambda} \leq \sum_{\left\{\lambda \notin \Gamma_{N}: \lambda \leq \kappa 5^{m+1}\right\}} d_{\lambda}=O\left(2^{m-N} 3^{N}\right) .
$$

Moreover the number of $\lambda \in \Gamma_{N}$ such that $\lambda \leq \kappa 5^{m}$ is $\sum_{N+1}^{m} 2^{m-j}$ from the 5 series and $\sum_{N+1}^{m} 2^{m-j-1}$ from the 6 series, giving a total that is $O\left(2^{m-N}\right)$. This implies

$$
\frac{c 3^{N}}{d_{\Lambda}} \sum_{\lambda \in \Gamma_{N}} 1=O\left(2^{m-N} 3^{N} d_{\Lambda}^{-1}\right)
$$

and substituting this and (22) into (21), along with $d_{\Lambda} \geq \frac{1}{2}\left(3^{m}-3\right)$ because $\Lambda \geq \kappa 5^{m-1}$, we have

$$
\left|\frac{1}{d_{\Lambda}} \log \operatorname{det} M_{\Lambda}-\int_{S G} \log f d \mu\right| \leq 4 \epsilon+c\left(\frac{3}{2}\right)^{N-m},
$$

which proves the first statement of the theorem.

For Hölder continuous $f$ and fixed $\Lambda$ we may now optimize the choice of $N$ as in the proof of Theorem 1 to obtain 19. 
Remark 2. Observe that in comparison with (14), the error in (19) is decaying at a slower rate. This is a consequence of the fact that, at the optimal $N$, the eigenfunctions that are not localized at scale $N$ make up a larger proportion (in terms of dimension) of the space $E_{\Lambda}$ than they do in the spaces $E_{\lambda}$ with $\lambda \approx \Lambda$.

\section{5. "Almost" EQUALly Distributed SEQUENCES}

As mentioned to in the Introduction, (3) can be translated into results on equally distributed sequences. In this section we shall prove an analogue of (3) on SG. This will be used to define the notion of "almost" equally distributed sequences on SG. We recall the definition of equally distributed sequences due to $\mathrm{H}$. Weyl, for which we refer to [6. Chapter 5].

Definition 1. Fix $K>0$. For each $n$ let $a_{1}^{(n)}, \ldots, a_{n+1}^{(n)}$ and $b_{1}^{(n)}, \ldots, b_{n+1}^{(n)}$ be sets of $n+1$ numbers from the interval $[-K, K]$. We say that the sets $\left\{a_{j}^{(n)}\right\}$ and $\left\{b_{k}^{(n)}\right\}, n \rightarrow \infty$, are equally distributed in the interval $[-K, K]$, if given any continuous function $F$ on $[-K, K]$ we have

$$
\lim _{n \rightarrow \infty} \frac{1}{n+1} \sum_{j=1}^{n+1}\left[F\left(a_{j}^{(n)}\right)-F\left(b_{j}^{(n)}\right)\right]=0 .
$$

We first consider an extension of the results proved in Section 3 . Recall that $M_{j}=P_{j}[f] P_{j}$, where $P_{j}$ is the orthogonal projection onto the eigenspace $E_{j}$ corresponding to a 5 or 6 -series eigenvalue $\lambda_{j}$ of $-\Delta$ with generation of birth $j$. Let $\left\{\sigma_{k}^{(j)}\right\}_{k=1}^{d_{j}}$ be the eigenvalues of $M_{j}$

Lemma 2. Let $f=\sum_{k=1}^{3^{N}} a_{k} \chi_{C_{k}}$ with all $a_{k}>0$ and let $m=\min _{k} a_{k}$, $M=\max _{k} a_{k}$. Let $F$ be continuous on $[m, M]$. Then

$$
\lim _{j \rightarrow \infty} \frac{1}{d_{j}} \sum_{k=1}^{d_{j}} F\left(\sigma_{k}^{(j)}\right)=\int_{S G} F(f(x)) d \mu(x) .
$$

Moreover there is a set of points $\left\{s_{k}^{(j)}\right\}_{k}^{d_{j}}$ in $S G$ such that $\left\{\sigma_{k}^{(j)}\right\}$ and $\left\{f\left(s_{k}^{(j)}\right)\right\}$ are "almost" equally distributed in $[m, M]$ when $j \rightarrow \infty$.

Remark 3. We use the term "almost" equally distributed because the above limit is computed along the subsequence $d_{j}$ of the positive integers.

Proof. Note from the proof of Lemma 1 that all eigenvalues of $M_{j}$ satisfy $m \leq \sigma_{k}^{(j)} \leq M$, and that each $a_{k}$ is an eigenvalue of $M_{j}$ with 
multiplicity $m_{j}^{N}$. The remaining eigenvalues form a set $\Upsilon_{j}$ with $\# \Upsilon_{j} \leq$ $\alpha_{j}^{N}$. Using the fact that $m_{j}^{N}=3^{-N} d_{j}^{N}$ we compute

$$
\begin{aligned}
\mid \frac{1}{d_{j}} & \sum_{k=1}^{d_{j}} F\left(\sigma_{k}^{(j)}\right)-\int_{S G} F(f(x)) d \mu \mid \\
& =\left|\frac{1}{d_{j}} \sum_{\Upsilon_{j}} F\left(\sigma_{k}^{(j)}\right)+\frac{m_{j}^{N}}{d_{j}} \sum_{k=1}^{3^{N}} F\left(a_{k}\right)-\int_{S G} F(f(x)) d \mu\right| \\
& \leq \frac{\alpha_{j}^{N}}{d_{j}}\|F\|_{L^{\infty}([m, M])}+\left(\frac{d_{j}^{N}}{d_{j}}-1\right) \int_{S G} F(f(x)) d \mu \\
& \leq \frac{2 \alpha_{j}^{N}}{d_{j}}\|F\|_{L^{\infty}([m, M])} .
\end{aligned}
$$

The last part of the result follows from approximating $\int_{\mathrm{SG}} F(f(x)) d \mu(x)$ with Riemann sums.

With this result we can prove the following extension of Theorem 1 Theorem 3. Let $f>0$ be continuous on $S G$ and $M=\max _{S G} f(x)$. If $F$ is continuous on $[0, \infty)$, then

$$
\lim _{j \rightarrow \infty} \frac{1}{d_{j}} \sum_{k=1}^{d_{j}} F\left(\sigma_{k}^{(j)}\right)=\int_{S G} F(f(x)) d \mu(x) .
$$

Moreover there is a set of points $\left\{s_{k}^{(j)}\right\}_{k}^{d_{j}}$ in $S G$ such that $\left\{\sigma_{k}^{(j)}\right\}$ and $\left\{f\left(s_{k}^{(j)}\right)\right\}$ are "almost" equally distributed in $\left[0,\|f\|_{L^{\infty}}\right]$ as $j \rightarrow \infty$.

Proof. Let $\delta>0$ be given and let $0<\epsilon<\delta$ be such that $|a-b| \leq \epsilon$ and $a, b \in[0, M]$ implies $|F(a)-F(b)|<\delta$. Take $f_{N}$ a simple function as in the proof of Theorem 1. We saw in that proof that the eigenvalues of $P_{j}[f] P_{j}$ that correspond to the eigenfunctions localized at scale $N$ are bounded below by $(1-\epsilon) a_{k}$ and above by $(1+\epsilon) a_{k}$. Writing $\rho_{k}^{j}$ for the eigenvalues of $P_{j}\left[f_{N}\right] P_{j}$ we have

$$
\begin{aligned}
\left|\frac{1}{d_{j}} \sum_{k=1}^{d_{j}} F\left(\sigma_{k}^{(j)}\right)-\int_{S G} F(f(x)) d \mu\right| \\
\leq\left|\frac{1}{d_{j}} \sum_{k=1}^{d_{j}} F\left(\rho_{k}^{(j)}\right)-\int_{S G} F\left(f_{N}(x)\right) d \mu\right|+\left|\frac{1}{d_{j}} \sum_{k=1}^{d_{j}}\left(F\left(\sigma_{k}^{(j)}\right)-F\left(\rho_{k}^{(j)}\right)\right)\right| \\
\quad+\left|\int_{S G}\left(F(f(x))-F\left(f_{N}(x)\right)\right) d \mu(x)\right| \\
\leq \frac{2 \alpha_{j}^{N}}{d_{j}}\|F\|_{L^{\infty}([m, M])}+\delta+\epsilon .
\end{aligned}
$$


This proves the first statement, and the second statement follows using Riemann sums as before.

More generally we have the following extension of Theorem [2, in which we denote the eigenvalues of $M_{\Lambda}$ by $\left\{\sigma_{k}^{(\Lambda)}\right\}_{k=1}^{d_{\Lambda}}$.

Theorem 4. Let $f>0$ be continuous on $S G$ and $F$ be continuous on $[0, \infty)$. Then

$$
\lim _{\Lambda \rightarrow \infty} \frac{1}{d_{\Lambda}} \sum_{k=1}^{d_{\Lambda}} F\left(\sigma_{k}^{(\Lambda)}\right)=\int_{S G} F(f(x)) d \mu(x) .
$$

Moreover there is a set of points $\left\{s_{k}^{(\Lambda)}\right\}_{k}^{d_{\Lambda}}$ in $S G$ such that $\left\{\sigma_{k}^{(\Lambda)}\right\}$ and $\left\{f\left(s_{k}^{(\Lambda)}\right)\right\}$ are "almost" equally distributed in $\left[0,\|f\|_{L^{\infty}}\right]$ as $\Lambda \rightarrow \infty$.

Proof. For $\delta>0$ and $N$ as in Theorem 3 we decompose the sum into terms corresponding to $\lambda \in \Gamma_{N}$ and $\lambda \notin \Gamma_{N}$ as in Theorem 2. For $\lambda \in \Gamma_{N}$ we estimate as in the proof of Theorem 3 , and follow the argument of Theorem 2 to find that the left and right sides of (23) differ by at most $2 \delta+O\left(2^{m-N} 3^{N}\right)$ where $\Lambda \approx 5^{m}$.

Remark 4 . The results proved here for SG should extend to other fractals on which localized eigenfunctions come to predominate in the spectrum as the eigenvalues increase. Sufficient symmetry conditions for the existence of high multiplicity eigenspaces with localized eigenfunctions were given in [2].

Acknowledgment. The authors are grateful to Victor Guillemin for suggesting that we investigate these questions.

\section{REFERENCES}

[1] M. T. Barlow, Diffusion on Fractals, in: Lectures Notes in Mathematics, Vol. 1690, Springer, Berlin, 1998.

[2] M. T. Barlow and J. Kigami, Localized eigenfunctions of the Laplacian on p.c.f. self-similar sets, J. London Math. Soc., 56 (1997), no. 2, 320-332.

[3] K. Dalrymple, R. S. Strichartz, and J. P. Vinson, Fractal differential equations on the Sierpinski gasket, J. Fourier Anal. Appl., 5 (1999), no. 2/3, 203-284.

[4] M. Fukushima and T. Shima, On a spectral analysis for the Sierpinski gasket, Potential Anal., 1 (1992), 1-35.

[5] B. Golinskii and I. Ibragimov, A limit theorm of G. Szegö, Math. USSR-Izv. 5 (1971), no. 2, 421-444.

[6] U. Grenander and G. Szegö, "Toeplitz forms and their applications," Second edition, Chelsea Publishing Co., New York, 1984.

[7] V. Guillemin and K. Okikiolu, Szegö theorems for Zoll operators, Math. Res. Lett. 3 (1996), 1-14.

[8] I. I. Hirschman, Jr. The strong Szegö limit theorem for Toeplitz determinants, Amer. J. Math. 88 (1966) 577-614. 
[9] M. Kac, Toeplitz matrices, translation kernels and a related problem in probability theory, Duke Math. J. 21, (1954), 501-509.

[10] J. Kigami, Harmonic calculus on p.c.f. self-similar sets, Trans. Amer. Math. Soc., 335 (1993), 721-755.

[11] J. Kigami, "Analysis on Fractals," Cambridge University Press, New York, 2001.

[12] A. Laptev and Y. Safarov, Szegö type limit theorems, J. Funct. Anal. 138 (1996), 544-559.

[13] K. Okikiolu, The analogue of the strong Szegö limit theorem on the 2- and 3-dimensional spheres, J. Amer. Math. Soc. 9 (1996), no. 2, 345-372.

[14] K. A. Okoudjou and R. S. Strichartz, Asymptotics of eigenvalue clusters for Schrödinger operators on the Sierpiński gasket, Proc. Amer. Math. Soc. 135 (2007), no. 8, 2453-2459.

[15] R. Rammal and G. Toulouse, Random walks on fractal structures and percoloration clusters, J. Physique Lett., 44 (1983), L13-L22.

[16] R. S. Strichartz, Analysis on fractals, Notices Amer. Math. Soc., 46 (1999), 1199-1208.

[17] R. S. Strichartz, Function spaces on fractals, J. Funct. Anal. 198 (2003), no. $1,43-83$.

[18] R. S. Strichartz, "Differential Equations on Fractals: a Tutorial," Princeton University Press, Princeton, NJ, 2006.

[19] G. Szegö, On certain Hermitian forms associated with the Fourier series of a positive function, Comm. Sém. Math. Univ. Lund [Medd. Lunds Univ. Mat. Sem.], (1952). Tome Supplementaire, 228-238.

[20] A. Teplyaev, Spectral analysis on infinite Sierpiński gaskets, J. Funct. Anal., 159:537-567, 1998.

[21] H. Widom, Szegö's theorem and a complete symbolic calculus for pseudodifferential operators, Seminar on Singularities of Solutions, Princeton Univ. Press, (1979), pp. 261-283.

Kasso A. Okoudjou, Department of Mathematics, University of MaryLAND, College Park, MD 20742-4015, USA

E-mail address: kasso@math.umd.edu

Luke G. Rogers, Department of Mathematics, University of ConNeCTiCUT, Storrs, CT 06269-3009, USA

E-mail address: rogers@math.uconn.edu

Robert S. Strichartz, Department of Mathematics, Malott Hall, Cornell University, IthaCA, NY 14853-4201, USA

E-mail address: str@math.cornell.edu 